Greenop, K., Peters, S.M., Bailey, H.D., Fritschi, L.E., Attia, J., Scott, R.J., Glass, D.C., De Klerk, N.H., Alvaro, F., Armstrong, B.K. \& Milne, E. (2013). Exposure to pesticides and the risk of childhood brain tumors. Cancer Causes and Control, 24(7), 1269-1278.

(C) Springer Science+Business Media Dordrecht 2013

This is pre-copy-editing, author-produced version of an article accepted for publication in Cancer Causes and Control, following peer review. The final publication is available at Springer via http://dx.doi.org/10.1007/s10552-013-0205-1. Please note: An erratum to this article is available at http://dx.doi.org/10.1007/s10552-014-0418-y.

This version was made available in the UWA Research Repository on $9^{\text {th }}$ of J uly 2014 , in compliance with the publisher's policies on archiving in institutional repositories.

Use of the article is subject to copyright law. 


\section{Exposure to pesticides and the risk of childhood brain tumors}

\section{Accepted by 'Cancer, Causes and Control’ 26/3/13}

Authors: Kathryn R. Greenop ${ }^{1}$, Susan Peters ${ }^{2}$, Helen D. Bailey ${ }^{1}$, Lin Fritschi ${ }^{2}$, John Attia ${ }^{3,4}$,

Rodney J. Scott ${ }^{4,5}$, Deborah C. Glass ${ }^{6}$, Nicholas H. de Klerk ${ }^{1}$, Frank Alvaro ${ }^{7}$, Bruce K. Armstrong ${ }^{8}$, Elizabeth Milne ${ }^{1}$

\section{Affiliations:}

1. Telethon Institute for Child Health Research, Centre for Child Health Research, University of Western Australia, Perth, Western Australia, Australia.

2: Western Australian Institute for Medical Research, University of Western Australia, Perth, Western Australia, Australia

3: Clinical Epidemiology and Biostatistics, University of Newcastle, Newcastle, New South Wales, Australia

4. Hunter Medical Research Institute, School of Biomedical Sciences, Faculty of Health, University of Newcastle, Newcastle, New South Wales, Australia.

5. Hunter Area Pathology Service, HNEHealth, Newcastle, New South Wales, Australia.

6: Department of Epidemiology and Preventive Medicine, Monash University, Melbourne, Victoria, Australia.

7: John Hunter Children's Hospital, Newcastle, New South Wales, Australia.

8: Sydney School of Public Health, University of Sydney, Sydney, New South Wales, Australia

\section{Author for correspondence:}

Elizabeth Milne

Telethon Institute for Child Health Research

PO Box 855 West Perth, Western Australia 6872, Australia

Telephone: +61 0894897756

Facsimile: +61 0894897700

Email: $\underline{\text { lizm@ichr.uwa.edu.au }}$ 


\section{Abstract}

Purpose

Previous research has suggested positive associations between parental or childhood exposure to pesticides and risk of childhood brain tumors (CBT). This Australian case-control study of CBT investigated whether exposures to pesticides before pregnancy, during pregnancy and during childhood were associated with an increased risk.

Methods

Cases were recruited from 10 paediatric oncology centres and controls by random-digit dialling, frequency matched on age, sex and state of residence. Exposure data were collected by written questionnaires and telephone interviews. Data were analysed by unconditional logistic regression. Results

The odds ratios (ORs) for professional pest control treatments in the home in the year before the index pregnancy, during the pregnancy, and after the child's birth were 1.54 (95\% confidence interval (CI): 1.07, 2.22), 1.52 (95\% CI: 0.99, 2.34) and 1.04 (95\% CI: 0.75, 1.43) respectively. ORs for treatments exclusively before pregnancy and during pregnancy were 1.90 (95\% CI: 1.08, 3.36) and 1.02 (95\% CI: 0.35, 3.00) respectively. The OR for the father being home during the treatment was 1.79 (95\% CI: 0.85, 3.80). The OR for paternal occupational exposure in the year before the child's conception was 1.36 (95\% CI: 0.66, 2.80). ORs for prenatal home pesticide exposure were elevated for low and high grade gliomas; effect estimates for other CBT subtypes varied and lacked precision.

Conclusions

These results suggest that preconception pesticide exposure, and possibly exposure during pregnancy, is associated with an increased CBT risk. It may be advisable for both parents to avoid pesticide exposure during this time.

Keywords: brain tumors, case-control studies, child, cancer, pesticides, insecticides 


\section{INTRODUCTION}

Childhood brain tumors (CBT) are the second most common type of childhood cancer after acute leukemia, and are often associated with poor survival and a high rate of post-treatment morbidity. The two established causes of CBT are genetic syndromes and ionising radiation, which together account for less than $5 \%$ of cases [1]. The early onset of most cases of CBT indicates that some predisposing or initiating events may occur before conception, during fetal life or in infancy. There is growing evidence that insecticide exposure may be involved in the etiology of cancer. Some insecticides such as bifenthrin, fipronil, permethrin and tetramethrin are rated by the US Environmental Protection Agency as possible or likely human carcinogens [2]. In Australia, these chemicals are common active constituents of termite barrier treatments or household surface insecticides [3]. Exposure of parents before or during pregnancy may have harmful effects on the germ cell or developing fetus, leading to DNA damage that may predispose to cancer development. Residues of some insecticides have been identified in umbilical cord blood, neonatal hair, and meconium after exposure during pregnancy [4,5]. After birth, children may be more susceptible to the harmful effects of pesticides than adults, as they have more actively dividing cells. Moreover, they may be more exposed because of increased contact with the ground and mouthing behavior during toddler years $[6,7]$.

Most previous studies have reported positive associations between maternal prenatal exposure to pesticides and risk of CBT [8-12]. Paternal exposure to various pesticides before and during the index pregnancy has also been associated with an increased risk [13-15]. None of these studies were able to separate parental exposure in the preconception period from that in the gestational period. A review of epidemiological studies published up to 2007 [16] concluded that prenatal parental exposure to pesticides in and around the home was associated with CBT risk. The evidence for an increased risk of CBT after childhood pesticide exposure has been less consistent, with some studies finding no association [10,17], one finding a positive association [18] and one finding a positive association but no evidence of a dose-response relationship [19].

The Australian Study of Childhood Brain Tumours (Aus-CBT) was a nationwide case-control study designed to investigate environmental and genetic risk factors for CBT. The aim of the current analysis was to examine whether exposure to pesticides before or during pregnancy, or during the child's life, was associated with an increased risk of CBT. For home exposure, professional pest control treatments were chosen as the exposure of interest as they are more likely to be accurately recalled by parents and are likely to be more highly concentrated, and therefore lead to higher exposure, than those for self-application. We also examined whether parental occupational exposure to pesticides before pregnancy was associated with childhood CBT; whether the specific 
timing, frequency, location, or type of pesticide exposure influenced any associations of home pesticide exposure with CBT.

\section{METHODS}

The design of Aus-CBT has been described in detail elsewhere [20]. Briefly, incident cases of CBT diagnosed between 2005 and 2010 were identified through all 10 pediatric oncology centers in Australia. Controls were recruited by national random digit dialling (RDD) during the same years and frequency matched to cases by age, sex and state of residence in a ratio of approximately 3:1. Cases and controls were only eligible for inclusion if they resided in Australia and had a biological parent available who could complete questionnaires in English. Between 2005 and 2010, we were notified of 794 CBT cases; 64 were ineligible (36 with no English-speaking parent, 23 nonresidents, five with no biological parent available). Of the 730 eligible cases, 568 (77.8\%) were invited to participate by a physician; with 162 eligible case families being deemed by the physician to be unsuitable to invite on medical or psychosocial grounds. Parents of 374 cases consented (65.8\% of invited, 51.2\% of eligible). Controls matched to CBT cases diagnosed in 2005 and 2006 were originally recruited as controls for our national case-control study of childhood acute lymphoblastic leukemia (Aus-ALL; 2003-2007), which used identical RDD recruitment methods [21,22]. Controls matched to CBT cases diagnosed in 2007-2010 were recruited prospectively. In total, 3,624 families eligible to be controls were identified, of whom 2,255 (62.2\%) agreed to participate. We recruited 1,467 of these children to the study according to age and sex frequencymatching requirements. Both studies were approved by the Human Research Ethics Committees at all participating hospitals.

\section{Data collection and exposure metrics}

Parents were mailed questionnaires that included questions about a range of potentially carcinogenic exposures. For domestic pesticide exposure, the mother's questionnaire asked if any professional pest control treatments had been carried out in the house or garden and, if so, whether they had occurred in the year before, during or after the pregnancy with the index child. After these questionnaires were returned, the mother was asked to complete a computer-assisted telephone interview (CATI) which sought details of pest control exposures, including the frequency, type of pest being targeted, location around or within the home, whether the parents (or the child if after the birth) were at home during the treatment, whether the bedrooms were treated and, if so, whether the parents (or the child if after the birth) slept in the bedroom within three days of the treatment. If a treatment occurred during the index pregnancy, the mother was asked which trimester. If the father 
lived at a different address from the mother in the year before pregnancy, he was asked about professional pest control treatments in his home.

For occupational exposure, mothers and fathers provided information about all jobs held for more than six months from the age of 15 to the year after the child's birth in the mailed questionnaires. For each job, subjects were asked the year started and finished, job title, employer, main tasks and hours worked per week. For jobs (or main tasks) involving potential exposure to agents of interest (e.g. exterminator, farmer), sets of additional detailed questions about tasks undertaken were selected. These job-specific modules were subsequently asked in computer assisted telephone interviews. Parental occupational exposure to pesticides (yes/no) was then assessed based on exposure rules created a priori that required active handling of pesticides. Subjects were classified as being exposed to pesticides in a certain occupation if they indicated they had mixed or applied any type of pesticide, or applied wood preservatives. Each parent answered questions about his or her occupational history only (i.e. no proxy report).

In addition to self-reported measures of socioeconomic status (parental education and household income), each participant's address was linked to an Australian Bureau of Statistics Census Collection District (CD). CDs were assigned a score for the area-based Index of Relative SocioEconomic Disadvantage (IRSD) [23].

Statistical analysis

Separate analyses were conducted for domestic exposure for each period of possible exposure: the year before pregnancy, during pregnancy, and after the child was born. Exposure was censored at the child's age at diagnosis (in years and months) for cases and the child's age at questionnaire return for controls; because controls were frequency matched, it was not possible to use the date of diagnosis for a specific corresponding case. For domestic exposure, the types of pests treated were grouped into three categories: termites, general insects and spiders, and rodents or birds. Subgroup analyse of the potential effects of treatment of bedrooms, whether the bedroom was slept in, and of exposure in each period exclusively were undertaken. Occupational exposure to pesticides was examined for use any time before the child's birth and in the year before conception.

Odds ratios and 95 percent confidence intervals (95\% CIs) were estimated using unconditional logistic regression in PASW Statistics 18 (IBM SPSS software Inc., New York). All models were adjusted for study matching variables - child's sex, age of diagnosis/recruitment, and State of residence, and variables meeting the empirical definition of confounding; that is, associated with both case/control status and with pesticide use among control subjects in any time period (assessed by visual comparison of distributions and erring on the side of inclusion). For household pesticide treatments confounding variables were maternal age group, birth year, child's ethnicity and parental 
education, and maternal pre-pregnancy folic acid supplement use; for paternal occupational exposures they were paternal age, birth year, child's ethnicity, parental education, and both maternal and paternal occupational exposure to diesel exhaust.

\section{RESULTS}

Basic information on professional pest control was available for 303 (81\%) case families and 981 $\underline{941}(67 \underline{64} \%)$ control families. Detailed information on professional pest control from the CATI was available for 288 (77\%) case families and 917 (63\%) control families. Information about occupational exposure to pesticides was available for 247 (66\%) case fathers and 302 (81\%) case mothers, 799 (54\%) control fathers and 941(64\%) control mothers.

Demographic and other characteristics of cases and controls who provided data are shown in Table 1. Most distributions were similar, but controls were slightly more likely than cases to be female, have a mother aged over 35 years and have European ethnicity. A higher proportion of controls than cases were recruited in 2005-2006, as controls from our national leukemia study were frequency matched to CBT cases diagnosed in those years. Cases and controls lived in areas with higher average IRSD scores than the Australian population as a whole. The mean IRSD scores were 1025.3 for case CDs, 1030.5 for control CDs, and 1006.0 for all Australian CDs (data not shown in tables).

The ORs for professional pest control treatments before pregnancy, during pregnancy and after the child's birth were 1.54 (95\% CI 1.07, 2.22), 1.52 (95\% CI: 0.99, 2.34) and 1.04 (95\% CI: 0.75, 1.43) respectively (Table 2). The ORs for treatments done in the first, second and third trimesters of pregnancy were 0.76 (95\% CI: 0.27, 2.15), 1.30 (95\% CI: 0.68, 2.50) and 2.13 (95\% CI: 0.99, 4.60) respectively (not shown in table). The ORs for professional pest control treatments before pregnancy only, during pregnancy only and during childhood only were 1.90 (95\% CI: 1.08, 3.36), 1.02 (95\% CI: 0.35, 3.00) and 0.84 (95\% CI: 0.56, 1.26) respectively (Table 2).

Since most parents only reported one treatment for pre-pregnancy and pregnancy exposures, it was not possible to examine dose-response associations in these periods or to compare timing of exposure during pregnancy within individuals. For exposures after the child's birth, there was no increase in risk with two or more treatments or evidence of dose-response (data not shown).

For all time periods, the ORs for treatments done inside and outside the house were similar to each other (data not shown); although most parents ( 70\%) who reported a treatment reported it being applied both inside and outside the house. Analysis by pest type revealed a higher OR for termites than for spiders and insects in the pre-pregnancy period (Table 2). The OR for termite treatment 
during pregnancy was close to the null, although there were very few such treatments. The ORs for both termite and general insect/spider treatments during childhood were close to the null. There were insufficient exposures to rodent/bird treatments to allow analysis in any period.

The OR for the father being at home during professional pest treatment in the year before pregnancy was 1.79 (95\% CI 0.85, 3.80) (Table 2). The ORs for the mother being at home were 1.13 (95\% CI $0.66,1.96)$ for the pre-pregnancy period and 1.53 (95\% CI 0.83, 2.82) for during pregnancy. The OR for the mother being at home during pest treatment in either of these periods was 1.23 (95\% CI: $0.75,2.01$ ) (not shown in table). The OR for the child being at home during the treatment was 1.63 (95\% CI: 1.02, 2.60)

The ORs for the parents' bedroom being treated for pests in the year before pregnancy, during pregnancy and in either period were (respectively): 1.56 (95\% CI 1.10, 2.43), 1.53 (95\% CI 0.92, 2.53) (Table 2) and 1.56 (95\% CI 1.04, 2.35) (not shown in table). There appeared to be no association with either parent sleeping in the treated bedroom before pregnancy, while the OR for the mother sleeping in the treated bedroom during pregnancy was 1.60 (95\% CI 0.96, 2.69) (Table 2). The OR for the mother sleeping in the bedroom before or during pregnancy was 1.45 (95\% CI 0.93, 2.27) (not shown in table). No associations were seen for the child's bedroom being treated or the child sleeping in the bedroom. Supplementary Table 1 shows the results of the corresponding analyses when adjusting only for frequency matching variables. Generally, the ORs for pest control treatment exposure seen in these minimally adjusted models are lower than those of the equivalent fully adjusted models in Table 2.

There was no appreciable increasing trend in ORs for household exposure to pesticide treatments before or during pregnancy with increasing age of the child at diagnosis/recruitment (Supplementary Table 2).

The ORs for low grade gliomas associated with home pesticide exposure before and during pregnancy were similar to those for CBT overall ( 1.5$)$, while those for high grade gliomas were somewhat higher for both periods: 2.99 (95\% CI 0.99, 9.02) and 4.58 (95\% CI 1.39, 15.14) respectively (Table 3). There was also some suggestion of increased risks for ependymomas (both periods), and embryonal tumours (during pregnancy) although the effect estimates for these subgroups were imprecise.

Relatively few fathers were occupationally exposed to pesticides before the child's birth (Table 4). The ORs for occupational exposure at any time before the child's birth and in the year before pregnancy were similar: 1.28 (95\% CI 0.81, 2.02) and 1.36 (95\% CI 0.66, 2.80) respectively. The 
latter OR changed little when the reference group comprised fathers unexposed to pesticides in the year before the pregnancy (OR 1.33, 95\% CI: 0.65, 2.74) (not shown in table). ORs for maternal occupational exposure were not estimated as only two case and 14 control mothers were exposed to pesticides at any time before the child's birth.

A higher proportion of controls than cases had missing data, and controls missing data had a lower average IRSD than those with data. Therefore, an imputation analysis was conducted in which the frequency of pesticide exposure among controls with missing data was imputed from that of participating controls in the same IRSD range. The model was adjusted for matching variables, child's year of birth group and IRSD as a continuous variable (the only variables available for controls missing questionnaire data), and run 50 times and the ORs averaged. The results from this imputation analysis were 1.36 (95\% CI: 1.00, 1.85), 1.38 (95\% CI: 0.96, 1.98) and 0.93 (95\% CI: $0.71,1.23)$ for home professional pesticide use before, during and after the pregnancy respectively (Supplementary Table 3).

\section{DISCUSSION}

Our results indicate that professional pest control treatments in the home during the year before the index pregnancy may be associated with an increased risk of CBT. These results are also suggestive of a positive association with pest control treatment during the pregnancy, but there were few exposed cases and the ORs were close to the null when households treated before the pregnancy or after the birth were excluded. The ORs for some CBT subtypes, particularly highgrade gliomas, were higher than those for CBT overall. There was some evidence that paternal occupational exposure to pesticides in the preconception period was associated with increased risk. On the other hand, we found little evidence that treatments done after the child's birth were associated with risk of CBT. ORs were elevated if the child was home during the treatment, but this may be a chance finding or a result of reporting bias given the generally null results for childhood exposure.

Very few other studies have specifically investigated pest control exposure in the preconception period; most have looked at exposures during pregnancy or in a specified period prior to the child's birth that included both the pregnancy and the period leading up to it. In a French study, Cordier and colleagues reported an OR of $1.8(95 \% \mathrm{CI}: 0.8,4.1)$ for home pesticide treatments during pregnancy [8], and Pogoda and colleagues reported an OR of 1.7 (95\% CI: 1.1, 2.6) for prenatal exposure to flea and tick pesticides in the US [10]. In a study conducted in the USA and Canada, Van Wijngaarden and colleagues [11] reported positive associations between paternal insecticide, herbicide and fungicide exposure with astrocytoma, and herbicide exposure with primitive 
neuroectodermal tumors (PNETs). Maternal insecticide and fungicide exposure was associated with astroctyoma but not PNETs. Another study reported a positive association for insecticide use by the father only, and only for tumors other than astrocytoma and PNET; exposures in the two years before the child's birth were examined, so both the pregnancy and preconception periods were included.[13] Two other studies examining maternal pesticide exposure during gestation found little evidence of an association [17,24].

An international case-control consortium also reported positive associations with parental agricultural and/or occupational pesticide exposures during pregnancy or in the month before pregnancy $[14,12,9]$. A Swedish cohort study examining paternal occupational exposures 2 - 26 months before the child's birth reported a positive association: RR 2.36 (95\% CI: 1.27, 4.39) [15]. A Norwegian cohort study that analysed census data reported a positive association with agricultural pesticide expenditure (used as a proxy for pesticide use), but the exposure window was broad (5 years) [25].

While we found little evidence of any association between childhood pesticide exposure and risk of CBT, four previous case-control studies have reported at least some evidence of associations with various types of home pesticide exposure to the child $[8,18,17,14]$; however, the findings of two of these studies were imprecise and based on small numbers $[18,17]$. Three others reported null results $[10,19,26]$; one of these studies, however, did report increased risk among children diagnosed between 0-4 years of age [10]. These differences in findings are likely to be at least partly due to the variation in exposures and time periods examined.

Overall, our findings are consistent with most previous studies that exposure of parents during the preconception and/or gestational period is positively associated with CBT risk.

Professional pest control treatments in the home during the preconception period are likely to result in exposure for both parents who reside in the home, and it is difficult to determine which parent's exposure may be more important. The OR for preconception exposure was elevated when the father was at home during treatment but not when the mother was at home. These results are suggestive that paternal exposure to pesticides is at least as important as maternal exposure. If this is the case, the most plausible biological mechanism would be damage to the male germ cell. Developing sperm may be damaged by oxidative stress resulting from environmental exposures [27], and there is evidence that pesticides can induce oxidative stress in the lymphocytes of pesticide workers [16,28] and genotoxicity in domestic users [29]. DNA damage in sperm caused by oxidative stress and possibly other mechanisms may initiate cancer development in the offspring, although there is uncertainty as to the precise mechanism involved and which pesticides are most likely to produce these effects. Potential mechanisms by which maternal preconception exposure 
may play a role are less clear. One possibility is that residual chemicals in the mother's tissues or in the household environment from preconception exposure affect the embryo during very early development.

Our results also suggest that professional termite treatments may pose greater risk than general insecticide treatments. In Australia, termite treatments can only be applied by licensed pest controllers[30] chemicals approved for termite treatments include bifenthrin, fipronil, deltamethrin, chlorpyrifos, permethrin and imidacloprid - all of which are also used for extermination of pests other than termites [3,31]. Arsenic trioxide, hexaflumuron, and chlorfluazuron are used exclusively for termite treatments $[3,31]$. Although our study is not able to identify the exact chemicals that parents were exposed to, it is possible that chemicals used for termite treatments may be more strongly associated with risk of CBT.

The strengths of this study are that it was population-based, with case recruitment from all 10 paediatric oncology centres in Australia, and that control recruitment was undertaken by national random-digit dialling using state-of-the-art methods. During the recruitment period, around 90\% of the Australian population had a landline telephone [32]. The exposure of interest - professional pest control treatments - was chosen as it is likely to be recalled more accurately than general pesticide use around the home, thus reducing exposure measurement error. In addition, we used telephone interviews conducted by staff blinded to case/control status to acquire detailed information about the nature, timing and location of home treatments and occupational exposure so that these conditions could be analysed. We were also able to distinguish between exposures in different time periods relating to conception, gestation, and after the child's birth.

This study also had some limitations. The participation fraction of both cases and controls was relatively low, and lower for controls. Also, mean IRSD, an area-based measure of socioeconomic status, was higher in participating controls than cases (although both were higher than the national average). The tendency for controls to be living in higher SES areas than cases introduces the possibility of selection bias, particularly if exposure is related to SES. We were unable to determine pesticide use among non-participants but, as there is no clear relationship between home pesticide use and SES among participating controls, any effect of selection bias is difficult to predict. There was a tendency for paternal occupational exposures to pesticides to be more common in lower SES families: any resulting selection bias may have led to some overestimation of odds ratios. The results of our quantitative imputation analysis were not materially different from the reported results, suggesting that non-response to questionnaires could not fully explain our findings. As in all case-control studies, recall bias caused by case parents' increased rumination about possible exposures may have led to pest control treatments being over-reported among cases 
relative to controls. Recall bias would tend to inflate the ORs, but it is unlikely to produce different results for different exposure periods; thus, we believe our findings are unlikely to be entirely attributable to recall bias. On the other hand, case mothers may underreport recognized harmful exposures due to social desirability or feelings of guilt. This is plausible, given that parents are likely to associate chemicals that can kill insects or other animals with potential harm to their children. However, this would tend to lead to underestimation of a positive association, rather than inflate it. There is likely to have been error in the reporting of exposure, since parents were asked to recall details of treatments that occurred up to 15 years previously. Use of professional pest control treatments as the exposure of interest aimed to minimize such error, and for most families the period of recall would have been seven years or less. The lack of trend in the magnitude of ORs across child age groups suggests that it is unlikely that estimates were biased by differential recall between cases and controls over time. It was not possible to determine the exact chemical components of the pesticides used, which may vary greatly with respect to potential carcinogenicity. As these details were not asked of parents, this type of error would apply to both cases and controls.

Occupational exposure may be subject to recall bias as case parents may consider past exposures more than control parents. However, mixing or applying pesticides are very specific tasks that people are likely to remember if a part of their job. It is considered that the frequency that is more problematic to recall [33]. We classified parents as being either exposed or non-exposed, which we considered to be less prone to differential misclassification. Limiting the assignment of pesticide exposure to active handling may have led to misclassification of subjects passively exposed to pesticides in their job, e.g. by re-entry into the treated crop or packaging. Information on these types of exposure was not available from the job-specific questionnaires. This misclassification was assumed to be non-differential to case/control status. Moreover, the effect on the risk estimates is likely to be minimal, as these potentially exposed subjects would be few in number and would have been misclassified as non-exposed.

In conclusion, our findings are consistent with a positive association between home pesticide use in the year before the index pregnancy and risk of CBT. The relationship with occupational exposure to pesticides is less clear but suggests a positive association. As CBT is relatively rare, future pooling of data from similar studies would enable more detailed investigation of these associations, including analysis of dose-response and tumor subtypes. However, given the consistency of our findings with those of most previous studies, the avoidance of pest control treatments by parents in the prenatal period would be advisable. 


\section{ACKNOWLEGEMENTS}

\section{Consortium Statement}

The Aus-CBT consortium conducted the study and the Telethon Institute for Child Health Research (TICHR), University of Western Australia, was the coordinating centre. Bruce Armstrong (Sydney School of Public Health, University of Sydney), Elizabeth Milne, Nicholas de Klerk, Carol Bower, Peter Dallas (TICHR), Frank van Bockxmeer (Royal Perth Hospital, University of WA), Rodney Scott and John Attia (University of Newcastle), Lin Fritschi (WA Institute for Medical Research), Lesley Ashton, Michelle Haber and Murray Norris (Children’s Cancer Institute Australia for Medical Research, Lowy Cancer Research Centre, UNSW), Margaret Miller (Edith Cowan University) and Judith Thompson (WA Cancer Registry) were the research investigators.

The authors acknowledge the contribution made by our clinical co-investigators who recruited and cared for study patients at each participating hospital: Nicholas Gottardo (Princess Margaret Hospital, TICHR); John Heath and Elizabeth Smibert (Royal Children’s Hospital, Melbourne); Peter Downie (Monash Medical Centre, Melbourne); Tim Hassell and Ross Pinkerton (Royal Children’s Hospital Brisbane); Maria Kirby (Women’s and Children’s Hospital, Adelaide); Stewart Kellie and Luciano dalla Pozza (Westmead Hospital); Frank Alvaro (John Hunter Hospital, Newcastle); Richard Cohn (Sydney Children’s Hospital) and John Dauberton (Royal Hobart Hospital).

The authors also acknowledge the Clinical Research Associates at each hospital, and the study coordinators: Jackie Mansour, Somer Dawson, Tamika Heiden, and Helen Bailey; and Peter Cosgrove for programming the estimation of supplement intake from the food frequency questionnaires.

Funding: The National Health and Medical Research Council (NHMRC) funded Aus-ALL (Grant number: 254539) and Aus-CBT (Grant number: 404089). Elizabeth Milne and Lin Fritschi were supported by NHMRC Fellowships and Helen Bailey was supported by NHMRC Post Graduate Scholarship 513934. Support for Rodney Scott was in part from NBN Children's Cancer Research Fund.

\section{Conflict of Interest Disclosure: The authors declare that they have no conflict of interest.}




\section{REFERENCES}

1. Baldwin RT, Preston-Martin S (2004) Epidemiology of brain tumors in childhood--a review. Toxicol Appl Pharmacol 199:118-131

2. U.S. Environmental Protection Agency (2009) Chemicals Evaluated for Carcinogenic Potential. Office of Pesticide Programs, U.S. Environmental Protection Agency, Washington, D.C.

3. Australian Pesticides and Veterinary Medicines Authority Public Chemical Registration Information System: PUBCRIS [http://services.apvma.gov.au/PubcrisWebClient/welcome.do] Last accessed 23 August 2012.

4. Whyatt RM, Barr DB, Camann DE, Kinney PL, Barr JR, Andrews HF, Hoepner LA, Garfinkel R, Hazi Y, Reyes A, Ramirez J, Cosme Y, Perera FP (2003) Contemporary-use pesticides in personal air samples during pregnancy and blood samples at delivery among urban minority mothers and newborns. Environ Health Perspect 111:749-756

5. Ostrea EM, Jr., Bielawski DM, Posecion NC, Jr., Corrion M, Villanueva-Uy E, Bernardo RC, Jin Y, Janisse JJ, Ager JW (2009) Combined analysis of prenatal (maternal hair and blood) and neonatal (infant hair, cord blood and meconium) matrices to detect fetal exposure to environmental pesticides. Environ Res 109:116-122

6. Moya J, Bearer CF, Etzel RA (2004) Children's behavior and physiology and how it affects exposure to environmental contaminants. Pediatrics 113:996-1006

7. Freeman NC, Jimenez M, Reed KJ, Gurunathan S, Edwards RD, Roy A, Adgate JL, Pellizzari ED, Quackenboss J, Sexton K, Lioy PJ (2001) Quantitative analysis of children's microactivity patterns: The Minnesota Children's Pesticide Exposure Study. J Expo Anal Environ Epidemiol 11:501-509

8. Cordier S, Iglesias MJ, Le Goaster C, Guyot MM, Mandereau L, Hemon D (1994) Incidence and risk factors for childhood brain tumors in the Ile de France. Int J Cancer 59:776-782

9. Efird JT, Holly EA, Preston-Martin S, Mueller BA, Lubin F, Filippini G, Peris-Bonet R, McCredie M, Cordier S, Arslan A, Bracci PM (2003) Farm-related exposures and childhood brain tumours in seven countries: results from the SEARCH International Brain Tumour Study. Paediatr Perinat Epidemiol 17:201-211

10. Pogoda JM, Preston-Martin S (1997) Household pesticides and risk of pediatric brain tumors. Environ Health Perspect 105:1214-1220.

11. van Wijngaarden E, Stewart PA, Olshan AF, Savitz DA, Bunin GR (2003) Parental occupational exposure to pesticides and childhood brain cancer. Am J Epidemiol 157:989997 
12. Holly EA, Bracci PM, Mueller BA, Preston-Martin S (1998) Farm and animal exposures and pediatric brain tumors: results from the United States West Coast Childhood Brain Tumor Study. Cancer Epidemiol Biomarkers Prev 7:797-802

13. Shim YK, Mlynarek SP, van Wijngaarden E (2009) Parental Exposure to Pesticides and Childhood Brain Cancer: U.S. Atlantic Coast Childhood Brain Cancer Study Environ Health Perspect 17:1002-1006

14. Rosso AL, Hovinga ME, Rorke-Adams LB, Spector LG, Bunin GR (2008) A case-control study of childhood brain tumors and fathers' hobbies: a Children's Oncology Group study. Cancer Causes Control 19:1201-1207

15. Feychting M, Plato N, Nise G, Ahlbom A (2001) Paternal occupational exposures and childhood cancer. Environ Health Perspect 109:193-196.

16. Infante-Rivard C, Weichenthal S (2007) Pesticides and childhood cancer: an update of Zahm and Ward's 1998 review. J Toxicol Environ Health 10:81-99

17. Leiss JK, Savitz DA (1995) Home pesticide use and childhood cancer: a case-control study. Am J Public Health 85:249-252.

18. Gold E, Gordis L, Tonascia J, Szklo M (1979) Risk factors for brain tumors in children. Am J Epidemiol 109:309-319

19. Schuz J, Kaletsch U, Kaatsch P, Meinert R, Michaelis J (2001) Risk factors for pediatric tumors of the central nervous system: results from a German population-based case-control study. Med Pediatr Oncol 36:274-282

20. Milne E, Greenop KR, Bower C, Miller M, van Bockxmeer FM, Scott RJ, de Klerk NH, Ashton LJ, Gottardo NG, Armstrong BK (2012) Maternal use of Folic Acid and Other Supplements and Risk of Childhood Brain Tumors Cancer Epidemiol Biomarkers Prev 21:1933-1941

21. Bailey H, Milne E, de Klerk N, Fritschi L, Bower C, Attia J, Armstrong B (2010) Representativeness of child controls recruited by random digit dialing. Paediatr Perinat Epidemiol 24:293-302

22. Milne E, Royle JA, Miller M, Bower C, de Klerk N, Bailey H, Van Bockxmeer F, Attia J, Scott RJ, Norris MD, Haber M, Thompson J, Fritschi L, Marshall G, Armstrong BK (2009) Maternal folate and other vitamin supplementation during pregnancy and risk of acute lymphoblastic leukemia in the offspring. Int J Cancer 126:2690-2699

23. Australian Bureau of Statistics (2008) Information Paper: An Introduction to Socio-Economic Indexes, 2006 Cat. no. 2039.0. Commonwealth of Australia.

[http://www.ausstats.abs.gov.au/Ausstats/subscriber.nsf/0/D729075E079F9FDECA2574170 011B088/\$File/20390_2006.pdf] Accessed 13 January 2012. 
24. Kuijten RR, Bunin GR, Nass CC, Meadows AT (1990) Gestational and familial risk factors for childhood astrocytoma: results of a case-control study. Cancer Res 50:2608-2612

25. Kristensen P, Andersen A, Irgens LM, Bye AS, Sundheim L (1996) Cancer in offspring of parents engaged in agricultural activities in Norway: incidence and risk factors in the farm environment. Int J Cancer 65:39-50

26. Howe GR, Burch JD, Chiarelli AM, Risch HA, Choi BCK (1989) An Exploratory Case-Control Study of Brain Tumors in Children. Cancer Res 49:4349-4352

27. Aitken RJ, Roman SD (2008) Antioxidant systems and oxidative stress in the testes. Oxid Med Cell Longev 1:15-24

28. Garaj-Vrhovac V, Zeljezic D (2000) Evaluation of DNA damage in workers occupationally exposed to pesticides using single-cell gel electrophoresis (SCGE) assay. Pesticide genotoxicity revealed by comet assay. Mutat Res 469:279-285

29. Lieberman AD, Craven MR, Lewis HA, Nemenzo JH (1998) Genotoxicity from domestic use of organophosphate pesticides. J Occup Environ Med 40:954-957

30. Commonwealth Scientific and Industrial Research Organisation (CSIRO) (2009) Fact sheet:

Termite or 'white ant treatment' and prevention. CSIRO, Australia.

http://www.csiro.au/en/Outcomes/Safeguarding-Australia/Termites/Termite-pestmanagement-and-control.aspx. Accessed 16 January 2013

31. Office of Chemical Safety; Australian Government Department of Health and Ageing (2007)

Termite Protection: Available treatments and hazard information about termiticides.

Commonwealth of Australia.

[http://www.health.gov.au/internet/main/publishing.nsf/Content/ocs-termite.htm] Accessed September 262012.

32. Australian Bureau of Statistics (2008) Western Australia Statistical Indicators, Sept 2008.Cat.

No: 1367.5. ABS, Commonwealth of Australia.

[http://www.abs.gov.au/AUSSTATS/abs@.nsf/DetailsPage/1367.5Sep\%202008] Accessed September 2012

33. Kersemaekers WM, Verheijen N, Kromhout H, Roeleveld N, Zielhuis GA (1998) Assessment of exposure to solvents among hairdressers: reliability of a classification scheme and questionnaire. Occup Environ Med 55:37-42 
Table 1: Demographics and birth characteristics of cases and controls in the Australian Study of Childhood Brain Tumours.

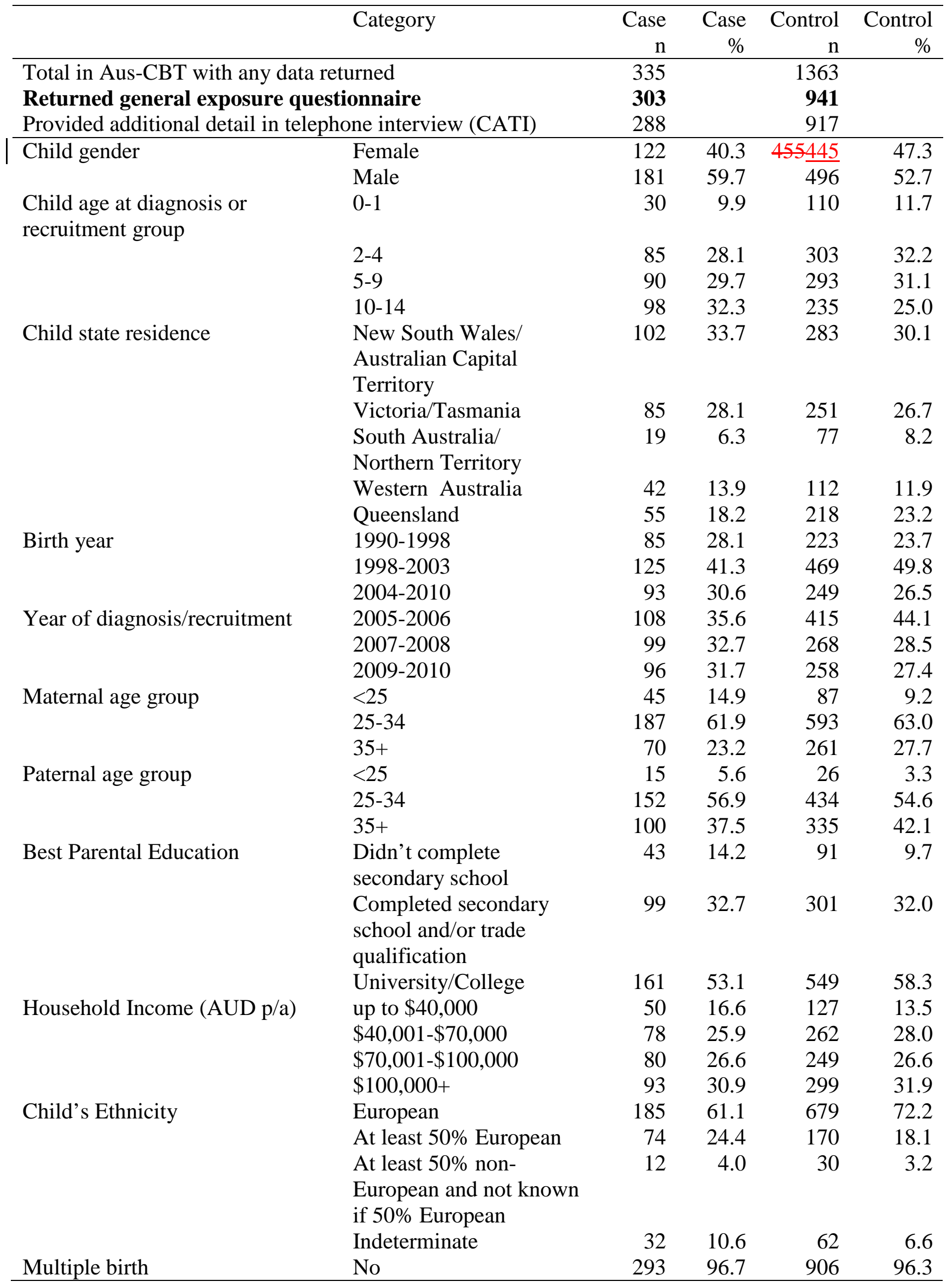




\begin{tabular}{rlrrrr}
\hline \multirow{3}{*}{ Birth defect } & Yes & 10 & 3.3 & 35 & 3.7 \\
& No & 289 & 95.4 & 903 & 96.0 \\
& Yes & 14 & 4.6 & 38 & 4.0 \\
\hline
\end{tabular}


Table 2: Professional pest control treatments in the home or garden and risk of childhood brain tumors

\begin{tabular}{|c|c|c|c|c|c|c|c|c|c|}
\hline & \multicolumn{9}{|c|}{ Time Period } \\
\hline & \multicolumn{3}{|c|}{ In the year before pregnancy } & \multicolumn{3}{|c|}{ During pregnancy } & \multicolumn{3}{|c|}{ After the child's birth ${ }^{1}$} \\
\hline & n case/cont & $\mathrm{OR}^{3}$ & $95 \%$ CI & n case/cont & $\mathrm{OR}^{3}$ & $95 \% \mathrm{CI}$ & n case/cont & $\mathrm{OR}^{3}$ & $95 \% \mathrm{CI}$ \\
\hline No pest control treatment ${ }^{4}$ & $170 / 548$ & 1.0 & Referent & $170 / 548$ & 1.0 & Referent & $170 / 548$ & 1.0 & Referent \\
\hline Any pest control treatment & $72 / 187$ & 1.54 & $1.07,2.22$ & $46 / 116$ & 1.52 & $0.99,2.34$ & $93 / 303$ & 1.04 & $0.75,1.43$ \\
\hline Any pest control in this period only & $23 / 46$ & 1.90 & $1.08,3.36$ & $5 / 18$ & 1.02 & $0.35,3.00$ & $44 / 165$ & 0.84 & $0.56,1.26$ \\
\hline \multicolumn{10}{|l|}{ Type of pest treated } \\
\hline Any termite treatment ${ }^{5}$ & $17 / 31$ & 2.17 & $1.12,4.19$ & $4 / 18$ & 1.06 & $0.33,3.41$ & $22 / 78$ & 1.00 & $0.59,1.72$ \\
\hline $\begin{array}{l}\text { Any general treatment for } \\
\text { spiders/insects }^{5}\end{array}$ & $55 / 156$ & 1.32 & $0.89,1.97$ & $31 / 86$ & 1.50 & $0.90,2.48$ & $80 / 260$ & 1.03 & $0.73,1.43$ \\
\hline Mother home during treatment & $23 / 81$ & 1.13 & $0.66,1.96$ & $19 / 49$ & 1.53 & $0.83,2.82$ & & & \\
\hline Father home during treatment & $12 / 29$ & 1.79 & $0.85,3.80$ & & & & & & \\
\hline Child home during treatment & & & & & & & $38 / 91$ & 1.63 & $1.02,2.60$ \\
\hline \multicolumn{10}{|l|}{ Location of Treatment } \\
\hline Bedroom treated $^{6}$ & $45 / 120$ & 1.56 & $1.01,2.43$ & $31 / 83$ & 1.53 & $0.92,2.53$ & $54 / 185$ & 0.97 & $0.65,1.44$ \\
\hline Mother slept in bedroom ${ }^{7}$ & $23 / 88$ & 1.19 & $0.69,2.06$ & $30 / 76$ & 1.60 & $0.96,2.69$ & & & \\
\hline Father slept in bedroom ${ }^{7}$ & $22 / 87$ & 1.16 & $0.66,2.03$ & & & & & & \\
\hline Child slept in bedroom ${ }^{7}$ & & & & & & & $45 / 169$ & 0.88 & $0.57,1.34$ \\
\hline
\end{tabular}

${ }^{1}$ These data are for children who are known to have been exposed within the censoring date. This analysis excludes children for whom information about the age of exposure was not available.

${ }^{2}$ From the year before the pregnancy to the censoring date.

${ }^{3}$ Model adjusted for matching variables (child's age, sex, State of residence), year of birth group, maternal age group, child's ethnicity, parental education, maternal pre-pregnancy folic acid supplement use.

${ }^{4}$ Reference level for all analyses is no pest control treatments from the year before the pregnancy to the censoring date.

${ }^{5}$ Termites and general insects/spiders mutually adjusted for each other

${ }^{6}$ Parent's bedroom before and during pregnancy, child's bedroom after birth.

${ }^{7}$ Slept in bedroom within 3 days of the treatment 
Table 3: Professional Pest Control treatments and risk of childhood brain tumors by tumor type

\begin{tabular}{|c|c|c|c|c|c|c|c|c|c|}
\hline & \multicolumn{3}{|c|}{ Year before pregnancy } & \multicolumn{3}{|c|}{ During pregnancy } & \multicolumn{3}{|c|}{ After birth } \\
\hline & n case/cont & $\mathrm{OR}^{1}$ & $95 \% \mathrm{CI}$ & n case/cont & $\mathrm{OR}^{1}$ & $95 \% \mathrm{CI}$ & n case/cont & $\mathrm{OR}^{1}$ & $95 \% \mathrm{CI}$ \\
\hline \multicolumn{10}{|l|}{ Low Grade Gliomas } \\
\hline No pest control & $80 / 548$ & 1.00 & Referent & $80 / 548$ & 1.00 & Referent & $80 / 548$ & 1.00 & Referent \\
\hline Any pest control & $33 / 187$ & 1.58 & $0.97,2.57$ & $22 / 116$ & 1.54 & $0.87,2.71$ & $44 / 303$ & 0.99 & $0.64,1.53$ \\
\hline \multicolumn{10}{|l|}{ High Grade Gliomas } \\
\hline No pest control & $15 / 548$ & 1.00 & Referent & $15 / 548$ & 1.00 & Referent & $15 / 548$ & 1.00 & Referent \\
\hline Any pest control & $7 / 187$ & 2.99 & $0.99,9.02$ & $6 / 116$ & 4.58 & $1.39,15.14$ & 7/303 & 1.25 & $0.45,3.43$ \\
\hline \multicolumn{10}{|l|}{ Embryonal Tumors ${ }^{2}$} \\
\hline No pest control & $40 / 548$ & 1.00 & Referent & $40 / 548$ & 1.00 & Referent & $40 / 548$ & 1.00 & Referent \\
\hline Any pest control & $18 / 187$ & 1.13 & $0.58,2.20$ & $14 / 116$ & 1.58 & $0.76,3.27$ & $28 / 303$ & 1.17 & $0.66,2.05$ \\
\hline \multicolumn{10}{|l|}{ Germ Cell Tumors } \\
\hline No pest control & $13 / 548$ & 1.00 & Referent & $13 / 548$ & 1.00 & Referent & $13 / 548$ & 1.00 & Referent \\
\hline Any pest control & $4 / 187$ & 0.91 & $0.22,3.67$ & $0 / 116$ & - & - & $3 / 303$ & 0.31 & $0.07,1.39$ \\
\hline \multicolumn{10}{|l|}{ Ependymomas } \\
\hline No pest control & $11 / 548$ & 1.00 & Referent & $11 / 548$ & 1.00 & Referent & $11 / 548$ & 1.00 & Referent \\
\hline Any pest control & 6/187 & 2.34 & $0.74,7.42$ & $3 / 116$ & 1.90 & $0.45,7.97$ & 6/303 & 1.32 & $0.44,3.99$ \\
\hline
\end{tabular}

${ }_{1}^{1}$ Adjusted for matching variables, birth year, maternal age, ethnicity, parental education, maternal pre-pregnancy folate supplementation.

${ }^{2}$ Includes medulloblastomas, Primitive neuroectodermal tumors (including pineal), atypical teratoid rhaboid tumors. 
Table 4: Paternal occupational exposure to pesticides and risk of childhood brain tumors

\begin{tabular}{llll}
\hline & $\mathrm{n}$ cases/controls & $\mathrm{OR}^{1}$ & 95\% CI \\
\hline Not exposed any time before the pregnancy & $210 / 705$ & 1.0 & Referent \\
Exposed any time before the pregnancy & $33 / 85$ & 1.28 & $0.81,2.02$ \\
Exposed in the year before the pregnancy & $13 / 29$ & 1.36 & $0.66,2.80$
\end{tabular}

\footnotetext{
${ }^{1}$ Adjusting for matching variables: child age, sex, State of residence, year of birth group, parental education, child's ethnicity, fathers age, maternal and paternal occupational diesel exhaust exposure.
} 
Supplementary Table 1: Professional pest control treatments in the home or garden and risk of childhood brain tumors (adjusting only for frequency matching variables).

\section{Time Period}

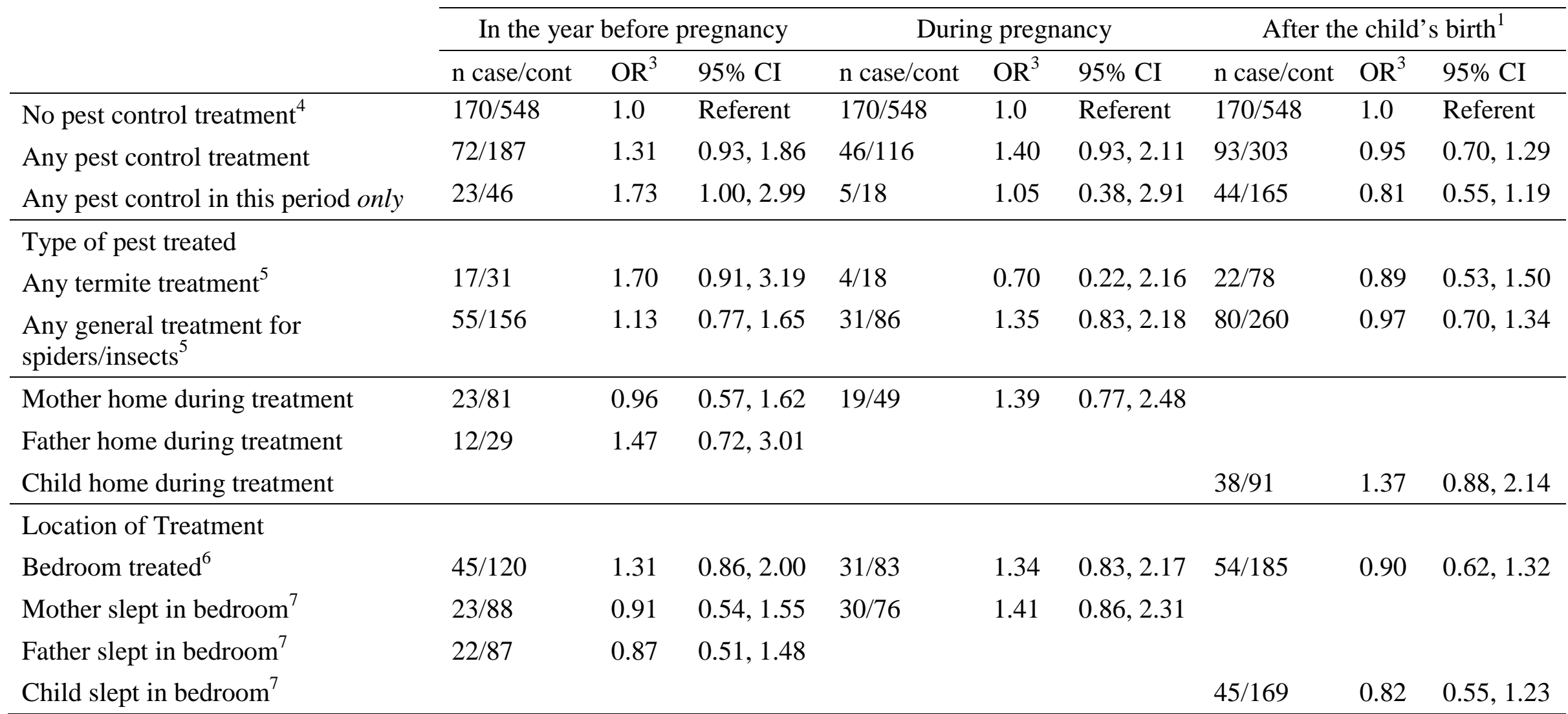

${ }^{1}$ These data are for children who are known to have been exposed within the censoring date. This analysis excludes children for whom information about the age of exposure was not available.

${ }^{2}$ From the year before the pregnancy to the censoring date.

${ }^{3}$ Model adjusted for matching variables (child's age, sex, State of residence).

${ }^{4}$ Reference level for all analyses is no pest control treatments from the year before the pregnancy to the censoring date.

${ }^{5}$ Termites and general insects/spiders mutually adjusted for each other

${ }^{6}$ Parent's bedroom before and during pregnancy, child's bedroom after birth.

${ }^{7}$ Slept in bedroom within 3 days of the treatment 
Exposure to pesticides and the risk of childhood brain tumors

Cancer Causes and Control

Authors: Kathryn R. Greenop, Susan Peters, Helen Bailey, Lin Fritschi, John Attia, Rodney J. Scott, Deborah C. Glass, Nicholas H. de Klerk, Frank Alvaro, Bruce K.

Armstrong, Elizabeth Milne

Author for correspondence:

Elizabeth Milne, Telethon Institute for Child Health Research, Email: lizm@ichr.uwa.edu.au 
Supplementary Table 2: Prenatal professional pest control treatment and risk of Childhood brain tumors stratified by child's age of diagnosis/recruitment.

\begin{tabular}{llllllll}
\hline & \multicolumn{3}{c}{ 12 months before pregnancy } & \multicolumn{3}{c}{ During pregnancy } \\
\hline Age & Any PPC & n case/cont & OR $^{1}$ & $95 \%$ CI & n case/cont & OR $^{1}$ & $95 \%$ CI \\
$0-1$ & No & $21 / 76$ & 1.00 & Ref & $21 / 76$ & 1.00 & Ref \\
& Yes & $6 / 22$ & 1.08 & $0.33,3.52$ & $5 / 14$ & 1.53 & $0.36,6.41$ \\
$2-4$ & No & $51 / 190$ & 1.00 & Ref & $51 / 190$ & 1.00 & Ref \\
& Yes & $21 / 62$ & 1.85 & $0.91,3.73$ & $14 / 47$ & 1.25 & $0.56,2.79$ \\
$5-9$ & No & $45 / 162$ & 1.00 & Ref & $45 / 162$ & 1.00 & Ref \\
& Yes & $25 / 59$ & 1.78 & $0.92,3.43$ & $18 / 34$ & 2.04 & $0.96,4.35$ \\
$10-14$ & No & $54 / 123$ & 1.00 & Ref & $54 / 123$ & 1.00 & Ref \\
& Yes & $22 / 44$ & 1.19 & $0.55,2.55$ & $10 / 21$ & 0.85 & $0.32,2.30$ \\
\hline \multicolumn{7}{l}{ Interaction term $p$-value } & \multicolumn{7}{c}{0.72} & & 0.42 \\
\hline
\end{tabular}

${ }^{1}$ ORs are adjusted for matching variables, year of birth group, maternal age group, parental education.

${ }^{2}$ Reference level is no PPC from 1 year prior pregnancy to censoring date.

Exposure to pesticides and the risk of childhood brain tumors

Cancer Causes and Control

Authors: Kathryn R. Greenop, Susan Peters, Helen Bailey, Lin Fritschi, John Attia, Rodney J. Scott, Deborah C. Glass, Nicholas H. de Klerk, Frank Alvaro, Bruce K. Armstrong, Elizabeth Milne

Author for correspondence:

Elizabeth Milne, Telethon Institute for Child Health Research, Email: lizm@ichr.uwa.edu.au 
Supplementary Table 3: Home and occupational pesticide exposure and the risk of childhood brain tumors, with controls with missing exposure data imputed.

\begin{tabular}{|l|l|l|l|}
\hline & \multicolumn{3}{|c|}{$\begin{array}{l}\text { Exposure \% from available controls with IRSD in } \\
\text { the same range as those controls missing data }\end{array}$} \\
\hline & $\begin{array}{l}\text { \% exposure applied } \\
\text { to missing controls }\end{array}$ & $\mathrm{OR}^{1}$ & $95 \%$ CI \\
\hline PPC pre pregnancy & $19.9 \%$ & 1.36 & $1.00,1.85$ \\
\hline PPC during pregnancy & $12.3 \%$ & 1.38 & $0.96,1.98$ \\
\hline PPC after birth & $32.8 \%$ & 0.93 & $0.71,1.23$ \\
\hline $\begin{array}{l}\text { Father occupational exposure any } \\
\text { time before birth }\end{array}$ & $10.9 \%$ & 1.29 & $0.86,1.94$ \\
\hline $\begin{array}{l}\text { Father occupational exposure in the } \\
\text { year before pregnancy }\end{array}$ & $3.8 \%$ & 1.47 & $0.78,2.76$ \\
\hline
\end{tabular}

${ }^{1}$ With 1467 controls, those with missing data imputed in R with ORs adjusted for child age, sex, state, yob group and IRSD.

PPC: professional pest control; IRSD: Index of relative socioeconomic disadvantage,

OR: odds ratio; CI: confidence interval.

Exposure to pesticides and the risk of childhood brain tumors

Cancer Causes and Control

Authors: Kathryn R. Greenop, Susan Peters, Helen Bailey, Lin Fritschi, John Attia, Rodney J. Scott, Deborah C. Glass, Nicholas H. de Klerk, Frank Alvaro, Bruce K. Armstrong, Elizabeth Milne Author for correspondence:

Elizabeth Milne, Telethon Institute for Child Health Research, Email: lizm@ichr.uwa.edu.au 
Erratum for: Exposure to pesticides and the risk of childhood brain tumors

Cancer Causes Control (2013) 24:1269-1278

DOI 10.1007/s10552-013-0205-1

Authors: Kathryn R. Greenop, Susan Peters, Helen D. Bailey, Lin Fritschi, John Attia, Rodney J. Scott, Deborah C. Glass, Nicholas H. de Klerk, Frank Alvaro, Bruce K.

Armstrong, Elizabeth Milne

After publication, it was noted by the authors that the dataset used for the analyses of occupational exposures in this study accidentally used incomplete occupational histories collected for 94 control fathers and 104 control mothers recruited in 2006, and these control parents were all treated as unexposed to occupational pesticides.

Results in Tables 1-3 and Supplemental Tables 1 and 2 (and the first 3 rows of Supplemental Table 3) of our published paper were unaffected by this error as they concerned household, rather than occupational pesticide exposure. Our conclusions regarding the association with household exposure to professional pest controls are consequently unchanged.

However, for occupational exposure, 18 control fathers and 2 control mothers were mistakenly classified as being unexposed to pesticides when they should have been classified as exposed (any time before the child's birth). The analyses presented in Table 4 and parts of Supplemental Table 3 in the published manuscript were re-run using the full dataset to ensure correct exposure assignment ( 5 case fathers and 4 control fathers were additionally excluded from revised occupational analysis due to genuinely missing data). The updated Table 4 and Supplemental Table 3 (occupational estimates only) are presented in this erratum.

Our original results for paternal occupational pesticide exposures were imprecise and we concluded that these results were less clear than for household exposure but suggestive of a positive association. The revised ORs for paternal occupational exposure to pesticides are attenuated and offer little or no evidence of an increased risk. Our imputation analysis (Supplemental Table 3) still shows that these results were unlikely to be affected by bias due to missing job histories. The sentence in the abstract concerning father's occupational exposure should now read "The OR for paternal occupational exposure in the year before the child's conception was 1.11 (95 \% CI: 0.55, 2.23).” There were still too few occupationally exposed mothers to analyse ( 2 cases/16 controls).

We offer our sincere apologies for any confusion we may have caused. 
Table 4: Paternal occupational exposure to pesticides and risk of childhood brain tumors

\begin{tabular}{|l|l|l|l|}
\hline & $\mathrm{n}$ cases/controls & $\mathrm{OR}^{\mathrm{a}}$ & $95 \%$ CI \\
\hline $\begin{array}{l}\text { Not exposed any time } \\
\text { before the pregnancy }\end{array}$ & $201 / 680$ & 1.0 & Referent \\
\hline $\begin{array}{l}\text { Exposed any time before } \\
\text { the pregnancy }\end{array}$ & $32 / 103$ & 1.07 & $0.68,1.68$ \\
\hline $\begin{array}{l}\text { Exposed in the year before } \\
\text { pregnancy }\end{array}$ & $13 / 37$ & 1.11 & $0.55,2.23$ \\
\hline
\end{tabular}

${ }^{a}$ Adjusting for matching variables: child age, sex, State of residence, year of birth group, parental education, child's ethnicity, fathers age, maternal and paternal occupational diesel exhaust exposure.

Supplementary Table 3: Occupational pesticide exposure and the risk of childhood brain tumors, with controls with missing exposure data imputed.

\begin{tabular}{|l|l|l|l|}
\hline & \multicolumn{3}{|c|}{$\begin{array}{l}\text { Exposure \% from available controls with IRSD in } \\
\text { the same range as those controls missing data }\end{array}$} \\
\hline & $\begin{array}{l}\text { \% exposure applied } \\
\text { to missing controls }\end{array}$ & OR & $95 \%$ CI \\
\hline $\begin{array}{l}\text { Paternal occupational exposure any } \\
\text { time before birth }\end{array}$ & $13.3 \%$ & 1.08 & $0.72,1.62$ \\
\hline $\begin{array}{l}\text { Paternal occupational exposure in } \\
\text { the year before pregnancy }\end{array}$ & $4.9 \%$ & 1.16 & $0.62,2.14$ \\
\hline
\end{tabular}

${ }^{\mathrm{a} W i t h} 1467$ controls, those with missing data imputed in $\mathrm{R}$ with ORs adjusted for child age, sex, state, yob group and IRSD. IRSD: Index of relative socioeconomic disadvantage, OR: odds ratio; CI: confidence interval. 